\title{
Providing Care for Pediatric Cancer Patients in COVID-19 Era; Rapid Response Recommendations from A Developing Country
}

\author{
Mahmoud Elzembely ${ }^{1,2}$, Yousra Abdelrahman ${ }^{2}$, Shady Fadel ${ }^{3}$, and Hanafy Hafez ${ }^{4}$ \\ ${ }^{1}$ Assiut University \\ ${ }^{2}$ South Egypt Cancer Institute \\ ${ }^{3}$ Alexandria School of Medicine \\ ${ }^{4}$ National Cancer Institute (NCI), Cairo University
}

May 11, 2020

Coronavirus disease 2019 (COVID-19) was first described in December 2019 in Wuhan, the capital of China's Hubei province. ${ }^{1,2}$. On March 11, 2020, WHO declared COVID-19 as a pandemic ${ }^{3}$. The first confirmed case of COVID-19 in Egypt was reported on February 14, 2020. As of May 10, 2020, there have been 8,964 confirmed cases, 2,002 recovered and 514 deaths $^{4}$.

\section{Management of Pediatric Cancer in the COVID-19 ERA}

Children with cancer are immunocompromised and are at higher risk of developing serious complications because of infection exposure. During COVID-19 pandemic, many major challenges face pediatric oncologist regarding the continuity of the anticancer therapy, hospital admission, infection protection precautions and expected critical complication post infection in pediatric cancer patients. This raises a question of how to organize pediatric cancer care in the COVID-19 ERA? We propose some critical recommendations focusing primarily on viral infection prevention and control strategies, avoid significant myelosuppression and decreasing the duration of hospitalization or the use of medical resources

\section{Our recommendations include:}

Screening and surveillance:

All patients and their care givers should be screened with epidemiological history and screening questionnaires according to the updated COVID-19 case definition at a well-equipped station better to be at facility entrance. Suspected cases should be immediately isolated in an area separate from other patients. Medical workers caring for isolated patients should not care for regular patients in the oncology ward.

Apply Hand and respiratory hygiene:

Hand and respiratory hygiene should be strictly applied for patients, care givers and health workers ${ }^{5}$. Proper personal protective equipment should be used according to WHO guidance ${ }^{6}$.

Apply principles of social distancing:

Principles of social distancing should be applied through isolating patients in separate rooms and if not possible arrange patients' beds with 2 meters distance at least, only one caregiver is allowed during hospital admission or in outpatient clinic, no visitors are allowed and distancing patients in the waiting area by at least 2 meters

Prioritize Pediatric Cancer Care: 


\section{For newly diagnosed cases:}

Patient should proceed with their diagnostic procedures without delay. Diagnostic procedures should be performed as an outpatient procedure unless otherwise required by the treating physicians.

\section{For patients on active curative treatment}

Treatment protocols need to be modified to decrease hospitalization and decrease the duration of neutropenia without survival impairment.

\section{Hematological Malignancies}

\section{Acute lymphoblastic leukemia:}

Patients are treated according to modified St. Jude total $\mathrm{XV}^{7}$ protocol. We propose:

1-Giving granulocyte colony stimulating factors (GCSFs) during induction chemotherapy if BMA at D19 is in complete remission and after induction chemotherapy before consolidation.

2-Delaying consolidation and giving weekly methotrexate (MTX)/ 6-mercaptopurine (MP) for 2 weeks as interim continuation if BMA at the end of induction is MRD negative

3-During consolidation, reduction of the $6 \mathrm{MP}$ dose by $50 \%$ with close assessment of total leucocytic counts.

4- During continuation: for low risk patients, omitting vincristine (VCR)/dexamethasone (DEX) pulses and use only MTX/6MP in the addition to re-induction weeks and for high risk patients, no change Lasparaginase and reinduction weeks, omitting weeks of cyclophosphamide/cytarabine and use MTX/6MP alternating with VCR/DEX. These modifications are based on randomized controlled study by Felice et al ${ }^{8}$.

\section{Acute Myeloid leukemia:}

Patients are treated according to modified St. Jude AML 02 protocol $^{9}$, we propose the following recommendations:

1. Induction chemotherapy: Omit etoposide so induction consists of Ara-C and Doxorubicin based on Medical research council AML 15 clinical trial ${ }^{10}$.

2. The use of G-CSF during induction chemotherapy 24 hours after chemotherapy and 48 hours before the next cycle of chemotherapy if BMA at D15 less than 5\%.

\section{Non-hodgkin lymphoma including mature B ALL:}

We recommend using growth factors pre-emptively 24 hours after completion of chemotherapy.

\section{Hodgkin lymphoma, classic type}

Patients are treated according to EuroNet-PHL-CI clinical trial. We propose the use of OEPA and COPDAC chemotherapy as multiple day regimens in the day care without admission

Solid Tumors:

\section{We recommend}

1. Use of growth factors pre-emptively 24 hours after completion of chemotherapy

2. Shift of chemotherapy protocols as a day care outpatient therapy if possible, without endangering patients with possible adverse effects as nephrotoxicity.

3. Delay all therapies beyond $1^{\text {st }}$ line therapy unless there is urgent clinical situation

4. Delay imaging procedures to monitor response to treatment unless urgent clinical condition

Patients on palliative care therapy

Palliative care patients need to be evaluated case by case as delay in palliative care may result in more suffering and subsequently more hospitalization. 


\section{We recommend the following:}

1. Patients on chemotherapy: Delay palliative chemotherapy and use less aggressive regimens

2. Patients on pain medications: Give patient a stock of pain medications for a longer time

3. Use of telemedicine to communicate with patients to determine the need for hospital visit

Follow up patients:

We recommend disease free patient not to visit the hospital, using telemedicine for communication with pediatric cancer survivors and exchange medical documents

\section{Conclusion}

We are trying to offer the optimal care for those critical patients, keeping their chances of cure from cancer and on the same way minimizing their exposure and risk for COVID19 pandemic infection and maintaining safe environment for both patients and healthcare providers.

\section{References}

1. Zhou, P., Yang, X. L., Wang, X. G., Hu, B., Zhang, L., Zhang, W., .. \& \& Chen, H. D. (2020). A pneumonia outbreak associated with a new coronavirus of probable bat origin. Nature , 579 (7798), 270-273.

2. Zhu, N., Zhang, D., Wang, W., Li, X., Yang, B., Song, J., .. \& Niu, P. (2020). A novel coronavirus from patients with pneumonia in China, 2019. New England Journal of Medicine .

3. "WHO Director-General's opening remarks at the media briefing on COVID19" (https://www.who.int/dg/speeches/detail/who-director-general-s-openingremarks-at-the-media-briefing-on-covid-19-11-march-2020). World Health Organization (WHO) (Press release). 11 March $2020 . \quad$ Archived (https://web.archive.org/web/20200311212521/https://www.who.int/dg/speeches/detail/whodirector-general-sopening-remarks-at-the-media-briefing-on-covid-19-11-march-2020) from the original on 11 March 2020. Retrieved 10 May 2020.

4. "https://www.worldometers.info/coronavirus/", Author: Worldometers.info, Publishing Date: 10 May, 2020, Place of publication: Dover, Delaware, U.S.A.

5. World Health Organization. (2020). Infection prevention and control during health care when COVID19 is suspected: interim guidance, 19 March 2020 (No. WHO/2019-nCoV/IPC/2020.3). World Health Organization.

6. World Health Organization. (2020). Rational use of personal protective equipment for coronavirus disease ( COVID-19) and considerations during severe shortages: interim guidance, 6 April 2020 (No. WHO/2019-nCov/IPC_PPE_use/2020.3). World Health Organization.

7. Pui, C. H., Relling, M. V., Sandlund, J. T., Downing, J. R., Campana, D., \& Evans, W. E. (2004). Rationale and design of Total Therapy Study XV for newly diagnosed childhood acute lymphoblastic leukemia. Annals of hematology , 83, S124-6.

8. Felice MS, Rossi JG, Gallego MS, et al.: No advantage of a rotational continuation phase in acute lymphoblastic leukemia in childhood treated with a BFM back-bone therapy. Pediatr Blood Cancer 57 (1):47-55, 2011

9. Rubnitz, J. E., Inaba, H., Dahl, G., Ribeiro, R. C., Bowman, W. P., Taub, J., .. \& Meshinchi, S. (2010). Minimal residual disease-directed therapy for childhood acute myeloid leukaemia: results of the AML02 multicentre trial. The lancet oncology , 11 (6), 543-552.

10. Burnett, A. K., Russell, N. H., Hills, R. K., Hunter, A. E., Kjeldsen, L., Yin, J., .. \& Milligan, D. (2013). Optimization of chemotherapy for younger patients with acute myeloid leukemia: results of the medical research council AML15 trial. Journal of clinical oncology , 31 (27), 3360-3368. 